\section{POSTER 25-78}

\section{The Accuracy of Prehospital Use of Pulse Oximetry Waveform Systolic Blood Pressure Measurement (POWS)}

\author{
Michael Addis, BA, EMT, *Brendan McCluskey, \\ Robert F. Lavery, BA, MICP, Bartholomew J. \\ Tortella, MTS, MD, FACS \\ New Jersey Trauma Center and University EMS, UMDNJ-New \\ Jersey Medical School, New Jersey
}

Purpose: Blood pressure (BP) in the prehospital setting is one of the most important diagnostic tools used by EMS providers. Conventional methods of palpation and auscultation can be time-consuming, and often are inaccurate due to the adverse working conditions encountered. Pulse Oximetry Waveform Systolic (POWS) BP measurement has been utilized successfully in emergency departments to monitor BP. The objective of this study was to compare the accuracy of field POWS with systolic BP measurements obtained by noninvasive, electronic BP measurement (N1BP), auscultation (AUSC), and palpation (PALP) in the prehospital environment.

Methods: BP measurements used for this study were obtained by N1BP (PROPAQ Model 102), AUSC, PALP and by observing the return of the waveform upon cuff deflation on the pulse oximeter. The order in which the readings were obtained, as well as the arm chosen for measurement were random.

Results: A total of 63 patients were sampled. Regression analysis provided significant correlations between the four methods utilized $(p<0.0001)$.

\begin{tabular}{lccc} 
& \multicolumn{3}{c}{ Correlation Coefficient $(\boldsymbol{r})$} \\
PROPAQ & POWS & PALP \\
POWS & 0.92 & - & - \\
PALP & 0.92 & 0.96 & - \\
AUSC & 0.89 & 0.92 & 0.93
\end{tabular}

Conclusions: POWS is a fast, easy, and accurate technique with which to measure systolic BP in the field. It may have special importance for noisy environments, moving vehicles, or for hypotensive or obese patients where conventional methods of auscultation or palpation may be difficult.

\section{POSTER 26-89}

\section{Impact Of Red Lights And Siren on Response And Transport Times in an Urban EMS System}

\author{
*Harinder S. Dhindsa, MD, Anthony McIntyre, \\ MD, Robert Bass MD, John Howell, MD, Edward \\ Thornton, NREMT-P \\ Georgetown and George Washington Universities, District of \\ Columbia, Emergency Medical Services, Washington, D.C.
}

Purpose: To determine if the use of lights and siren in an urban environment decreases response time to the scene, and from the scene to the hospital.

Methods: A prospective, observational study measured response times from dispatch to scene, and from scene to hospital. "Chase unit" ambulances were paired with in-service ambulances. Data were collected during various hours of the day and night from all days of the week. When in-service units were dispatched, chase units allowed one minute to start responding, then proceeded without lights or siren to the incident using the same route. When patients were transported, the same procedure was followed from incident location to the hospital. Times and distances were recorded by independent observers on the response unit and by the driver on the chase unit.

Results: Time and distances for 37 incident responses and 27 transports were measured. The response times for the in-service units $(5.0 \pm 3.03 \mathrm{~min}$; $95 \% \mathrm{CI}$ : $4.0-6.0$ $\min ; p<0.01)$ were different than were those for the chase units $(8.6 \pm 4.6 \mathrm{~min} ; 95 \%$ CI: $7.0-10.1 \mathrm{~min}$; $p<0.01)$. The scene-to-hospital time for the in-service units (6.4 $\pm 3.0 \mathrm{~min}$.; $95 \%$ CI: $5.2-7.6 \mathrm{~min}$; $p=0.003$ ) also were shorter than for the chase units $(9.4 \pm 4.1 \mathrm{~min}$; $95 \%$ CI: 7.8-11.1 min; $p=0.003)$. The combined times for the response units ( $10.9 \pm 4.9 \mathrm{~min}$; $95 \%$ CI: $9.1-12.8 \mathrm{~min}$; $p=0.0003$ ) also were shorter than were those for the chase units $(17.3 \pm 7.3 \mathrm{~min}$; $95 \% \mathrm{CI}$ : $14.5-20.0 \mathrm{~min} ; p=$ 0.0003)].

Conclusions: The difference of $3.6 \mathrm{~min}$ in response time may be clinically significant in patients requiring cardiopulmonary resuscitation or other ALS care. The difference in combined times of 6.3 min similarly may be clinically significant for critically ill patients. 